\title{
Risk Assessment of Warship (Automatic) Navigation Plan Based on SVM
}

\author{
Hui Song
}

Dalian Naval Academy of the PLA, Dalian, Liaoning, China

\begin{abstract}
The automatic control of naval vessels is based on the navigational plan, and the navigational safety depends on the scientific nature of the plan, so the risk assessment of the navigational plan is very important. At present, the commonly used evaluation methods are too subjective. In this paper, an evaluation model based on support vector machine is proposed, and the empirical study is carried out with historical data. The results show that the evaluation model based on support vector machine has good self-learning ability and feature extraction ability, which can provide reference for the risk assessment of naval vessels.
\end{abstract}

\section{Introduction}

Promoting the "one belt and one way" construction has surpassed the one dimensional marine globalization in the past and launched the engine of the balanced development of the world economy. With the expansion of national ocean interests, the frequency and scope of naval vessel navigation are constantly expanding. The safety of naval vessel navigation is an important guarantee for the implementation of military or nonmilitary activities. Navigation risk involves many factors, complex relations and difficult integration. As an important part of warship navigation, navigation plan is of great significance for ensuring the safe navigation of ships. Therefore, it is important to make risk decisions for warship navigation plans. Traditional risk decisionmaking requires a lot of manual analysis by industry experts and analysts. Most of the data are extracted by subjective judgment. Most of the risk assessment methods have the shortcomings of large randomness, low accuracy and can not be generalized. Therefore, a set of navigational plans is established for naval vessels. The risk assessment method that is effective and convenient to use is of high practical value. Support Vector Machine (SVM) was first proposed by Corinna Cortes and Vapnik in 1995. It has many unique advantages in solving small sample, nonlinear and high-dimensional pattern recognition, and can be extended to other machine learning problems such as function fitting. SVM is based on the theory of statistical learning. It uses $\mathrm{VC}$ dimension theory and structural risk minimization principle to find the best compromise between the complexity of the model and the learning ability according to the limited sample information in order to obtain the best generalization ability. It is an effective machine learning algorithm developed in recent years to deal with nonlinear problems. At present, there are few samples of factors affecting the safety of naval vessels and formation navigation, and the general data mining technology is based on unlimited samples. Therefore, a risk assessment method based on SVM is proposed to evaluate many naval plans.

\section{SVM model and its evaluation steps}

\subsection{SVM classification algorithm}

SVM is developed from the optimal classification plane under linear separable conditions, and its basic idea is shown in Figure 1.

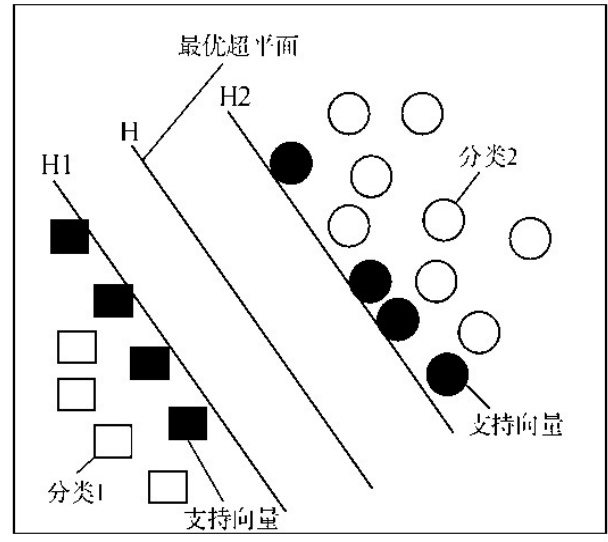

Figure 1. Optimal classification hyper plane.

In the graph, solid points and hollow points represent two types of samples, $H$ is the classification line, $H_{1}, H_{2}$ is the nearest sample from the classification line and parallel to the classification line, the distance between them is called classification interval. The optimal classification line requires that classification not 
only separates the two classes correctly (the training error rate is 0 ), but also maximizes the classification interval. The classification line equation is:

$$
w x+b=0
$$

Formula: $w$ is the weight vector and $b$ is the threshold value.

After normalization, the linear separable sample set $\left(x_{i}, y_{i}\right), i=1,2, \cdots, n, x \in R, y_{i} \in\{-1,1\}$ satisfies:

$$
y_{i}\left(w x_{i}+b\right)-1 \geq 0
$$

At this time, the classification interval is $\frac{2}{\|w\|}$, and the maximum interval is equivalent to making $\|w\|$ minimum. Satisfaction (2) and the classification surface which minimizes $1 /\left(2\|w\|^{2}\right)$ is called the optimal classification surface, and the training sample points on $H_{1}, H_{2}$ are called support vectors. By using Lagrange optimization method, the above optimal classification surface problem can be transformed into a dual problem, that is, under the constraints $\sum_{i=1}^{n} y_{i} \alpha_{i}=0$ and $\alpha_{i} \geq 0(i=1,2, \cdots, \mathrm{n})$, the maximum value of the following functions can be obtained for $\alpha_{i}$.

$$
F(\alpha)=\sum_{i=1}^{\mathrm{n}} \alpha_{i}-\frac{1}{2} \sum_{i, j=1}^{\mathrm{n}} \alpha_{i} \alpha_{j} y_{i} y_{j}\left(x_{i}, x_{j}\right)
$$

In the formula, $\alpha_{i}, \alpha_{j}$ is the Lagrange multiplier corresponding to the sample. Formula (3) has unique solution for the problem of optimization of two functions under inequality constraints. It is easy to prove that only a part of $\alpha_{i}$ is not zero in the solution, and the corresponding sample is support vector. The optimal classification function obtained by solving the above problem is

$$
f(x)=\operatorname{sgn}(w x+b)=\operatorname{sgn}\left[\sum \alpha_{i} y_{i}\left(x_{i} x\right)+b\right]
$$

For a linearly separable case, a relaxation term $\xi_{i} \geq 0$ can be added to the classification line equation:

$$
y_{i}\left(w x_{i}+b\right)-1+\xi_{i} \geq 0
$$

For nonlinear problems, the nonlinear transformation can be transformed into a linear problem in a highdimensional space, and then the space is transformed to find the optimal classification surface. According to the theory of functional theory, a kernel function $K\left(x_{i}, x_{j}\right)$ which satisfies Mercer condition can be used to realize the linear classification of a nonlinear transformation without knowing the form of transformation, without increasing the computational complexity. At this point, the objective function formula becomes

$$
F(\alpha)=\sum_{i=1}^{\mathrm{n}} \alpha_{i}-\frac{1}{2} \sum_{i, j}^{\mathrm{n}} \alpha_{i} \alpha_{j} y_{i} y_{j} K\left(x_{i}, x_{j}\right)
$$

And the corresponding classification function also becomes

$$
f(x)=\operatorname{sgn}(w x+b)=\operatorname{sgn}\left[\sum \alpha_{i} y_{i} K\left(x_{i}, x\right)+b^{*}\right]
$$

In the formula, $b^{*}$ is the threshold value of classification, which can be obtained by any one support vector or by taking the median value of any onedimensional support vector in the two classes.

\subsection{Evaluation steps of SVM}

(1) Set up a set of index system to express the risk level of warship navigation plan.

(2) Establish an evaluation grade, and take a certain number of implemented plans as samples in the past navigation plans.

(3) Select the appropriate kernel function and determine the corresponding parameters.

(4) Using the training samples, the decision support vector and related values are obtained by learning and training, and the parameters of the training model are adjusted by validating the training samples.

(5) Predict the overall risk level of the navigation plan according to the attribute value of the risk index of the navigation plan to be evaluated, and provide strong support for the risk response and risk management of the navigation plan.

\section{Empirical evaluation}

According to the procedure of SVM evaluation, this paper makes an empirical analysis on the risk assessment

\begin{tabular}{|c|c|}
\hline Two level index & First level index \\
\hline \multirow{4}{*}{$\begin{array}{l}\mathrm{U1} \\
\text { Environmental } \\
\text { risk }\end{array}$} & U11 Meteorology \\
\hline & U12 Route \\
\hline & U13 Electromagnetic \\
\hline & U14 Other ships \\
\hline \multirow{2}{*}{ U2 Ship risk } & U21 Equipment quality \\
\hline & U22 Loading weight \\
\hline \multirow{4}{*}{$\begin{array}{l}\text { U3 Personnel } \\
\text { risk }\end{array}$} & U31 Navigation technology \\
\hline & U32 Experience \\
\hline & U33 Personnel psychology \\
\hline & U34 Health \\
\hline \multirow{2}{*}{ 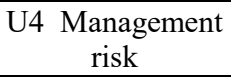 } & U41 The warship manages \\
\hline & U42 Port and formation manage \\
\hline
\end{tabular}
of naval plan. The concrete steps are as follows:

(1) In reference [1,2], a risk assessment index system for ship navigation plan is constructed, as shown in Table 1.

Table 1. Risk assessment index system for warship navigation plan. 
(2) Establish the evaluation level and obtain the data of nine projects (all of which are actual data obtained in escort or joint exercise) for pre-processing, as shown in Table 2. Among them: plan $1 \sim 6$ is training sample; plan $7 \sim 9$ is validation sample; plan 10 is sample to be evaluated. The risk level is 1, 2, and 3 respectively, indicating high, general and low risk.

(3) The RBF (Radial Basis Function) function is the most stable kernel function in the process of systematic research on planning risk and SVM, so this paper chooses this function as the kernel function of the risk assessment model. After many tests, the parameter variables are initially determined: $T=2$, which means taking $\mathrm{RBF}$ function; $C=100$, which represents the core parameters of C-SVC algorithm.

(4) Use the validated samples to verify the accuracy. Then we analyze and evaluate the risk of ship sailing plan. Using the data of Table 2, the model is obtained by SVM algorithm, and the model file train model is generated. Then the model is substituted into the verification data to verify. After operation, it is found that the accuracy of the result is exactly $100 \%$. Therefore, the model obtained through training data has good prediction ability.

(5) Plan 10 enters the evaluation stage after being certified, and the data are shown in Table 2. Using the trained model, the data of Plan 10 is calculated and the result shows that the risk level of the plan is 3 , belonging to the project with low risk.
In view of the characteristics of limited sample of risk data of naval ship navigation plan, a risk assessment model of naval ship navigation plan is proposed by using SVM method. The model can make full use of statistical information and expert opinions about risk factors of existing plans and minimize $\mathrm{VC}$ dimension by means of nonlinear mapping and maximizing classification boundary. On the basis of minimizing the empirical risk, the confidence risk can be minimized to minimize the structural risk. The implicit mechanism between the risk factors of the navigation plan and their influencing factors can be found and the comprehensive evaluation of the whole plan risk can be obtained.

In order to verify the superiority and inferiority of SVM evaluation, this paper attempts to use the same samples as the above to use neural network and fuzzy comprehensive evaluation method to evaluate. It is found that neural network has a problem of over-learning in small sample data processing, which makes the evaluation results more disorderly and does not get ideal results. When calculating weights, the whole evaluation process is complicated and subjective because of the need for experts to score, and it is not implemented at last. The SVM method has better objectivity, accuracy and effectiveness because of its self-learning and feature extraction ability.

Table 2. Training data for warship navigation plan.

\begin{tabular}{|c|c|c|c|c|c|c|c|c|c|c|c|c|c|}
\hline \multirow{2}{*}{ Plan } & \multicolumn{4}{|c|}{ U1 } & \multicolumn{2}{|c|}{ U2 } & \multicolumn{4}{|c|}{ U3 } & \multicolumn{2}{|c|}{ U4 } & \multirow{2}{*}{$\begin{array}{l}\text { Risk } \\
\text { level }\end{array}$} \\
\hline & U11 & U12 & U13 & U14 & U21 & U22 & U31 & U32 & U33 & U34 & U41 & U42 & \\
\hline 1 & 0.9 & 0.3 & 0.9 & 0.4 & 0.6 & 0.4 & 0.6 & 0.5 & 0.5 & 0.6 & 0.4 & 0.3 & 1 \\
\hline 2 & 0.8 & 0.2 & 0.9 & 0.3 & 0.5 & 0.4 & 0.5 & 0.6 & 0.6 & 0.4 & 0.3 & 0.4 & 1 \\
\hline 3 & 0.6 & 0.6 & 0.7 & 0.6 & 0.8 & 0.6 & 0.8 & 0.8 & 0.6 & 0.6 & 0.6 & 0.8 & 2 \\
\hline 4 & 0.5 & 0.7 & 0.6 & 0.7 & 0.7 & 0.7 & 0.7 & 0.6 & 0.7 & 0.7 & 0.8 & 0.6 & 2 \\
\hline 5 & 0.6 & 0.7 & 0.7 & 0.7 & 0.6 & 0.7 & 0.8 & 0.7 & 0.6 & 0.7 & 0.7 & 0.8 & 2 \\
\hline 6 & 0.3 & 0.9 & 0.3 & 0.8 & 0.9 & 0.9 & 0.8 & 1 & 1 & 0.8 & 0.9 & 0.8 & 3 \\
\hline 7 & 0.8 & 0.4 & 0.7 & 0.6 & $\begin{array}{l}0.8 \\
\end{array}$ & 0.3 & 0.7 & 0.6 & 0.5 & 0.5 & $\begin{array}{l}0.4 \\
\end{array}$ & 0.4 & 1 \\
\hline 8 & 0.3 & 0.9 & 0.3 & 0.8 & 0.9 & 0.9 & 0.8 & 1 & 1 & 0.8 & 0.9 & 0.8 & 3 \\
\hline 9 & 0.5 & 0.7 & 0.6 & 0.7 & 0.7 & 0.7 & 0.7 & 0.6 & 0.7 & 0.7 & 0.8 & 0.6 & 2 \\
\hline 10 & 0.4 & 0.9 & 0.2 & 0.9 & 0.8 & 0.9 & 0.7 & 0.9 & 0.9 & 0.9 & 0.8 & 0.9 & 3 \\
\hline
\end{tabular}

\section{Concluding remarks}

The risk assessment of naval vessel navigation plan is of vital importance to the accomplishment of naval mission, and is one of the main decision-making reference elements to determine the overall combat capability of naval vessels. Based on the comprehensive analysis of the influencing factors in the process of naval planning, this paper puts forward an evaluation index system of risk factors including environmental risk, naval ship risk, personnel risk and management risk. Based on SVM theory and its tools, this paper constructs the risk assessment process of naval vessel navigation plan, and carries out an empirical study combining with the actual data in the past. The solution to the risk analysis problem of naval vessel navigation plan characteristics (small sample size, high accuracy requirements, sensitive to the environment) is put forward, so as to solve the problem of naval vessel navigation plan. The decision analysis in navigation planning provides strong support and effective guidance.

\section{References}

1. $\mathrm{Hu}$ Shiping, Fang Quanggen, Qiao Guimin, $\mathrm{Xu}$ Bomin. Risk Analysis and Risk Control of Large Ships Navigation [J]. China Navigation, 2006 (3): 34-38.

2. Li Kan. Research on Risk Management of Weapon Equipment Development Projects [M]. Beijing: National Defense Industry Press, 2011: 76-147.

3. Zhou Jing, Song Hui, Li Tie, Li Minghai. A SVMbased rudder fault diagnosis method [J]. Military Automation, 2012 (4): 56-58.

4. Zhou Jing, Song Hui. Research on ship motion fitting method based on radial basis function neural network 
[J]. Computer and digital engineering, 2015 (9): 1588-1591.

5. Li Kan. Construction of risk management model for high-tech R\&D projects based on integrated approach [J]. China Science and Technology Forum, 2006 (6): 32-35.
6. Li Kan. Optimal design of equipment development scheme based on PDPC-EMV model [J]. Journal of Naval Aeronautical Engineering College, 2013, 28 (4): 447-450. 